\title{
PROSPECTS FOR MULTI-TEV TWO-BEAM LINEAR COLLIDERS*
}

\author{
Ronald D. Ruth \\ Stanford Linear Accelerator Center, Stanford, CA, USA
}

\begin{abstract}
Recent work at CERN and SLAC has opened the possibility of the development of concrete designs for electron positron linear colliders that have a center of mass energy substantially above $1 \mathrm{TeV}[1,2]$. These designs are based on high gradient, normal conducting acceleration with the power provided by an auxiliary beam that is efficiently accelerated in a fully loaded, low frequency linac. This type of power source offers a flexibility to develop linear collider designs that have a wide range of parameters. In particular, the choice of frequency can be made without regard to the availability of high power RF sources, at least up to about $30 \mathrm{GHz}$. This paper explores possible linear collider designs taking into account limits on acceleration gradient and beambeam effects. The study shows that electron positron linear colliders have an energy reach far in excess of 1 $\mathrm{TeV}$. In particular we show that an X-band linear collider powered with conventional sources might be upgraded using two-beam techniques to an energy far above $1 \mathrm{TeV}$. Thus, the linear collider offers a platform for continued exploration at the energy frontier of High Energy Physics.
\end{abstract}

\section{INTRODUCTION}

During the past decades High Energy Physics has taken great advantage of various new generations of accelerators as they have moved upwards on the energy frontier. Electron positron circular colliders have gone through several generations and have achieved an increase in energy reach of about a factor of 100 . This has been possible because our community has built on each generation and used it has the parent and teacher of the next generation. The culmination of e+e- circular collider energy frontier accelerators has been the outstanding success of LEP at CERN. Circular colliders have moved now to the Luminosity/Factory frontier with KEKB and PEP-II to do precision physics.

Proposals for electron-positron linear colliders have at their roots the single parent accelerator, the SLC at SLAC. The next round of proposals reach for a center of mass in the range of $1 / 2$ to $1 \mathrm{TeV}$, a factor of five to ten beyond the SLC.

The question that is addressed in this paper is whether or not we can build on this basis to provide an even further increase to multi-TeV linear colliders. If this is true, the electron positron linear collider could provide a platform to move onto precision physics at the multi $\mathrm{TeV}$ scale. Before proceeding with the energy issue it is useful to first discuss the luminosity.

*Work supported by DOE Contract DE-AC03-76F00515.

\section{LUMINOSITY}

The largest jump in all approaches to linear colliders is the luminosity. The desired luminosity is four orders of magnitude greater than the SLC luminosity. However, the $1 \mathrm{TeV}$ designs are building on the hard won success of the SLC, experiments at the Final Focus Test Beam and the ultra low emittance obtained in the KEK ATF. This experimental base has discovered a key feature that permeates the approach to obtaining high luminosity. In order to preserve the emittance and achieve the necessary small spots, it is critical that we pay attention to the interaction of the trajectory and emittance or beam size. Experience with the SLC, FFTB and KEK ATF has shown that beam based alignment is critical. To achieve stable beams feedback is also required. Special steering techniques, such as Dispersion Free Steering, which take into account the interaction of the trajectory with the projected beam size, are essential. The critical ingredients are a stable, precise set of instrumentation for beam position and beam size measurements. This data combined with the techniques for trajectory compensation with emittance preservation make the jump in luminosity at the next generation linear collider possible. However, it is important to note that in order to achieve the even higher luminosity at a multi $\mathrm{TeV}$ collider, it will be necessary to have experience with the $1 / 2$ to $1 \mathrm{TeV}$ linear collider.

\section{ENERGY}

Before discussing acceleration it is useful to recognize that all linear accelerators behave like transformers. Power from the grid (or co-generation plant) is transformed to a high-energy, pulsed, low-current electron/positron beam. Multi $\mathrm{TeV}$ linear colliders require high-gradient acceleration. The acceleration gradient sets the length scale, much like the superconducting magnet field set the length scale for the LHC. The power required for high gradient acceleration must be compressed and converted to RF to accelerate the beam. For conventional approaches this is done by the combination of modulators, klystrons, and RF pulse compression systems. Two-Beam RF power generation is envisioned for Multi $\mathrm{TeV}$ linear colliders because it provides frequency-independent energy compression. A two-beam power source can provide power at frequencies where there are no other sources. The 'Drive Beam' discussed later in this paper provides the low loss intermediate energy storage similar to the RF compression system for conventional systems. 


\section{HIGH GRADIENT ACCELERATION}

Historically, there has been hope that higher frequency RF systems can intrinsically support higher gradients. The NLC and CLIC designs have been based on this and on early experimental results that showed high gradients in short structures that required relatively low power to achieve high gradient. However, recent results have shown that although surface field plays a critical role, other aspects of the structure such as group velocity or cell position in the structure also appear to play a significant role. Finally, in structures with a very large number of breakdowns, the RF properties have been affected.

The status of the experimental breakdown studies at NLCTA is discussed in detail in these proceedings [3]. The details of the development of structures for testing and also simulations of breakdown are given in Refs [48]. Experimental results are coming in rapidly, but a summary of the situation is as follows:

1. Early short structures at X-Band have reached acceleration gradients of about $120 \mathrm{MV} / \mathrm{m}$. The record is held by a CERN X-band structure $30 \mathrm{~cm}$ long.

2. Full-length high group velocity structures $1.8 \mathrm{~m}$ long were conditioned up to about $70 \mathrm{MV} / \mathrm{m}$ and could run around $50 \mathrm{MV} / \mathrm{m}$ but showed phase shifts due to the extensive number of breakdowns.

3. The first half of these long high group velocity structures were damaged significantly and the second half were completely undamaged. These parts of the structure have nearly the same surface field.

4. The second half of the long structure was cut off and fitted with a new short front end and conditioned rapidly up to about $70 \mathrm{MV} / \mathrm{m}$ and could run steadily with little damage.

5. Several additional structures with $5 \%$ and $3 \%$ group velocity have now been tested and all show rapid conditioning above $70 \mathrm{MV} / \mathrm{m}$ and stable running near that level.

6. Most breakdowns take place at or near the input couplers for the low group velocity structures.

7. An additional testing program has been started to examine the possibility of using standing wave structures.

For the purposes of this paper we assume that the gradient studies for NLC will be successful. This requires that traveling wave structures run well at about $70 \mathrm{MV} / \mathrm{m}$. It requires that a standing wave structures run well at about $55 \mathrm{MV} / \mathrm{m}$.

There is presently little experience at higher frequency, although tests at CERN suggest that higher gradient may be possible at higher frequency. At X-band experimental results show no fundamental limits up to about 100 $\mathrm{MV} / \mathrm{m}$. For the remainder of this paper we assume that after some development a future X-band structure might reach stable running at a loaded gradient of about 94 $\mathrm{MV} / \mathrm{m}$. For a standing wave structure this would be the unloaded gradient as well while a traveling wave structure would have to be conditioned to an acceleration field in excess of $100 \mathrm{MV} / \mathrm{m}$.

If higher gradients are realized (or if extended length is possible), it is interesting to ask whether or not it is possible to use two-beam acceleration to upgrade the energy of an X-Band linear collider. Before addressing this question it is useful to discuss Two Beam acceleration.

\section{TWO-BEAM ACCELERATION}

The basic concept of two-beam acceleration is rather simple. In Figure 1 you see idea illustrated with example parameters. A high-current beam, tightly bunched at the operating frequency or a sub harmonic, is decelerated by a low-impedance decelerator structure. The resulting output RF is transferred in a waveguide to a high-gradient accelerating structure where it is used to accelerate the low-current, high-energy beam. In the example shown the drive beam is decelerated by $1.5 \mathrm{MeV}$ each meter while the main beam is accelerated by $93 \mathrm{MeV}$ each meter. The combination of decelerating and accelerating structures acts like a transformer moving the energy stored in the drive beam to the main beam. Depending on the final desired energy, the drive beam must be replaced after it loses about $90 \%$ of its energy to maintain its stability during deceleration [2].

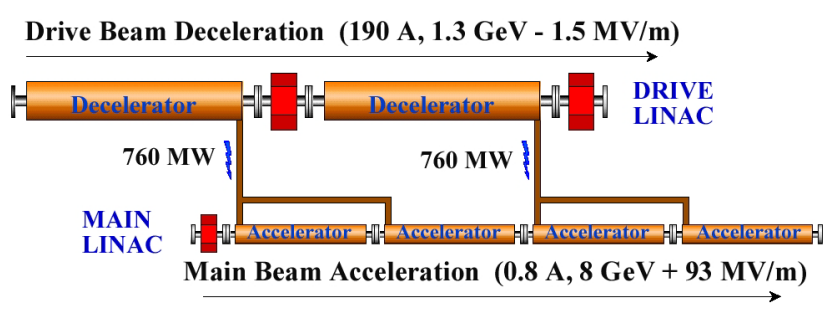

Figure 1: The Basic Concept of Two-Beam Acceleration

The creation of the drive beam is a key problem for two-beam schemes because it must be done efficiently with reliable technology. Recently, a new technique was introduced which uses a low frequency normal conducting linac to accelerate the drive beam. After acceleration the drive beam pulse structure is modified using rings that serve to compress the energy into pulses appropriate for $\mathrm{RF}$ production at high frequency. This technique is described in Refs. 1 and 2.

In a previous paper we introduced two new aspects to the drive beam creation process and the concept of a twobeam linear collider [9]. First, the drive beam can be accelerated in multiple passes through the drive beam linac. This recirculation reduces the number of klystrons but increases the pulse length of each. Second, these same power sources are used to power a higher gradient main beam injector accelerator after they have finished accelerating the drive beam. An overall layout that shows a two-beam system with recirculation is shown in Figure 2. 


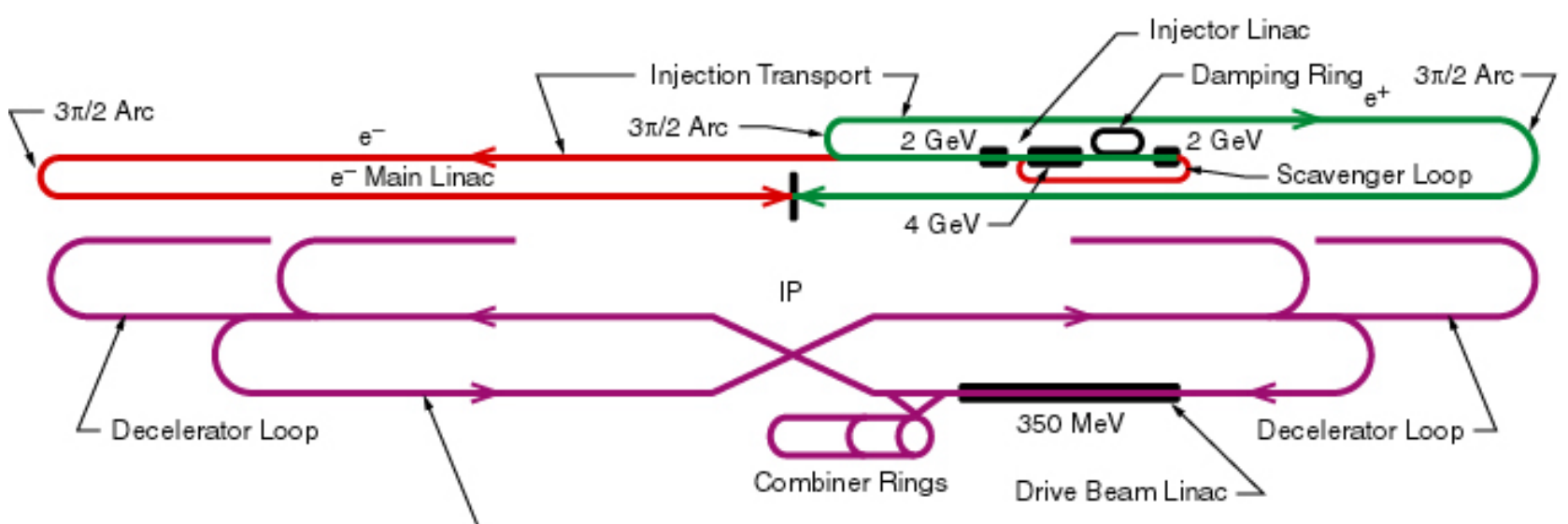

Drive Beam Recirculation Loop

Figure 2: Schematic Layout of a Two-Beam Linear Collider with Recirculation and Central injector. The upper beam lines are for the main beam while the lower ones are for the drive beam. All long beam lines are in the same tunnel. The vertical scale is expanded for clarity. The injector linac sits beside the drive beam linac. View animation here.

To understand Figure 2 it is useful to trace a complete two-beam cycle as follows (see also animation):

1. First accelerate a long-pulse drive beam in a fully loaded L-band linac. The drive beam pulse length is twice the length of the high gradient linac.

2. Recirculate the beam to accelerate in four passes trading off the number of klystrons with pulse length.

3. After final acceleration use combiner rings and RF deflection to interleave bunches $(2 \times 4 \times 4=16)$.

4. This compresses the long pulse beam into six shorter pulses and increases the bunch frequency by 16 .

5. Distribute pulses to decelerator. After compression, the pulses are separated by twice the length of the section that they will power.

6. Transfer power to the high gradient accelerator to accelerate the high-energy beam. As in Fig 1 the drive beam decelerates while the main beam is accelerated. After each section the depleted drive beam is dumped while another arrives at the correct time and phase to take over the acceleration.

While the use of the drive beam klystrons to power the injector is interesting for two beam systems built from scratch, it will not be considered for upgrades because the injector is presumed to exist.

\section{UPGRADES BEYOND 1 TEV}

For the purposes of this exercise we assume that an $\mathrm{X}$ band linear collider with the NLC design is working at 1 $\mathrm{TeV}$. The structures discussed earlier need to run at about $70 \mathrm{MV} / \mathrm{m}$ for traveling wave structures and at about 55 $\mathrm{MV} / \mathrm{m}$ for standing wave structures. The loaded gradient in each case is $55 \mathrm{MV} / \mathrm{m}$ and the geographic gradient is about $50 \mathrm{MV} / \mathrm{m}$. These parameters are consistent with present NLC designs. Let us assume that the structures will support a gradient of $94 \mathrm{MV} / \mathrm{m}$ loaded in a stable running configuration. This is not required of the NLC 1 $\mathrm{TeV}$ design and would require development beyond the present state of the art at X-band. However, this assumption is not unreasonable considering the experiments showing gradients in excess of $100 \mathrm{MV} / \mathrm{m}$ and given the rapid progress of the development of high gradient designs. On the other hand, a similar upgrade could be presented which keeps the NLC gradient but extends the length. This approach will not be presented because of the potential advantage of the evolution of the linear collider energy without substantial new construction.

The upgrade proposed here seeks to use the existing infrastructure and RF power as much as possible while adding a separate two-beam power source to increase the gradient. This hybrid approach seems awkward, but it permits an adiabatic transition to a two-beam linear collider of even higher energy as we discuss below.

The first upgrade uses a two-beam system to supply the power of $2 / 3$ of the structures of the linear collider which redirecting the existing power from the conventional system into one third of the structures. This is illustrated in Figure 3. Table 1 shows the parameters for the linear collider at the higher beam energy. As you can see from this table, the luminosity increases by about a factor of three while the energy increases to $1.7 \mathrm{TeV}$. The repetition rate has been dropped by a factor of two, so that the factor of three increase in power for RF only results in a $50 \%$ increase in the site power required for RF. The charge per bunch has been allowed to increase proportional to the gradient in order to keep the same beam loading to maintain high efficiency. The damping rings for this design have not been upgraded from those for the NLC, and we have assumed that the emittance dilution will be the same for the higher energy linac because of the higher gradient. The beam-beam effects are more severe as expected at higher energy. However, it may be possible to make design trade-offs which yield nearly the same luminosity but with smaller beam-beam effects. 

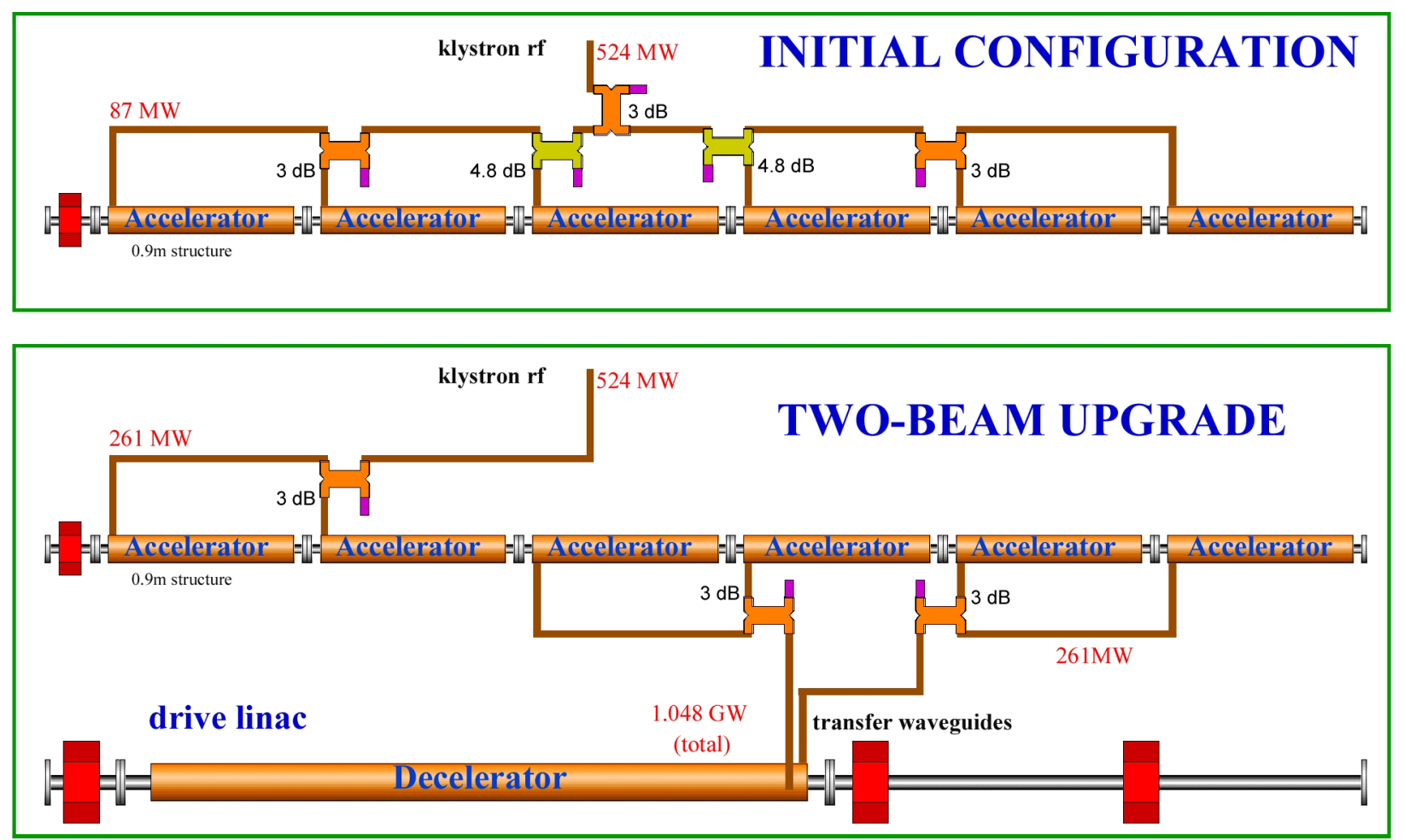

Figure 3: The initial configuration shows nominal parameters for the NLC operating at $1 \mathrm{TeV}$. For the two-beam upgrade the power from the klystrons is routed into two structures while a new two-beam system is installed to provide the power for the remaining two thirds of the structures.

Table 1: Parameters for NLC and Two-Beam Upgrade

\begin{tabular}{|l|r|r|r|}
\hline \multicolumn{3}{|c|}{ NLC Luminosity Parameters } & Upgrade \\
\hline & Stage 1 & Stage 2 & TB-NLC \\
\cline { 2 - 4 } CMS Energy $(\mathrm{GeV})$ & 500 & 1000 & 1700 \\
Luminosity $\left(\mathbf{1 0}^{\mathbf{3 3}}\right)$ & $\mathbf{2 0}$ & $\mathbf{3 4}$ & $\mathbf{9 4}$ \\
Repetition Rate $(\mathrm{Hz})$ & 120 & 120 & 60 \\
Bunch Charge $\left(\mathbf{1 0} 0^{\mathbf{1 0}}\right)$ & $\mathbf{0 . 7 5}$ & $\mathbf{0 . 7 5}$ & $\mathbf{1 . 3 5}$ \\
Bunches/RF Pulse & 190 & 190 & 190 \\
Bunch Separation $(\mathrm{ns})$ & 1.4 & 1.4 & 1.4 \\
Eff. Gradient $(\mathbf{M V} / \mathrm{m}$ & $\mathbf{5 0 . 2}$ & $\mathbf{5 0 . 2}$ & $\mathbf{7 8}$ \\
Injected $\gamma_{\mathrm{x}} / \gamma_{\mathrm{y}}\left(10^{-8}\right)$ & $300 / 2$ & $300 / 2$ & $300 / 2$ \\
$\gamma_{\mathrm{x}}$ at IP $\left(10^{-8} \mathrm{~m}-\mathrm{rad}\right)$ & 360 & 360 & 360 \\
$\gamma_{\mathrm{y}}$ at IP $\left(\mathbf{1 0} \mathbf{0}^{-8} \mathrm{~m}-\mathrm{rad}\right)$ & $\mathbf{3 . 5}$ & $\mathbf{3 . 5}$ & $\mathbf{3 . 5}$ \\
$\beta_{\mathrm{x}} / \beta_{\mathrm{y}}$ at IP $(\mathrm{mm})$ & $8 / 0.10$ & $10 / 0.12$ & $15 / 0.12$ \\
$\sigma_{\mathrm{x}} / \sigma_{\mathrm{y}}$ at IP $(\mathbf{n m})$ & $\mathbf{2 4 5} / \mathbf{2 . 7}$ & $\mathbf{1 9 0} / \mathbf{2 . 1}$ & $\mathbf{1 8 0} / \mathbf{1 . 6}$ \\
$\sigma_{\mathrm{z}}$ at IP $($ um $)$ & 110 & 110 & 110 \\
Yave & 0.11 & 0.29 & 1.04 \\
Pinch Enhancement & 1.43 & 1.49 & 1.6 \\
Beamstrahlung dB $(\%)$ & 4.7 & 10.2 & 31 \\
Photons per e+/e- & 1.2 & 1.3 & 2.3 \\
Linac Length $(\mathrm{km})$ & 6 & 12 & 12 \\
\hline
\end{tabular}

\section{DRIVE BEAM ACCELERATOR}

The Drive Beam Accelerator accelerates a long pulse high current beam in order to store all the energy necessary to accelerate the main beam. The drive beam parameters for the NLC X-band upgrade are shown in Table 2. The accelerator is powered by an L-band RF system. In this example we have not used recirculation for acceleration, the first half of the drive beam powers one linac while the second half powers the other. Recirculation can be used to decrease the number of drive beam structures and klystrons while increasing the drive beam RF power pulse width.

After acceleration the drive beam is compressed by the combiner ring complex to achieve a total 22 drive beam pulses each with 16 times the current. These are distributed, 11 to each linac, with the appropriate delay to power the main beam. The principle is identical to the animation and figure except that there is no recirculation; the first part of the path forms the delay necessary for the drive beam timing.

\section{UPGRADE TO 2.5 TEV (22.8 GHZ)}

The upgrade just described seems like a lot of effort to increase the energy. An alternative would be to modify the conventional system to achieve higher power via higher power klystrons or more pulse compression with longer klystron pulses. The primary reason for a twobeam upgrade is that allows the possibility to move to two-beam systems for even higher energy.

To illustrate this idea consider the drive beam parameters in the second column of Table 2 and also the beam parameters in Table 3 . The parameters in the second column of Table 2 show the drive beam necessary 
for a linear collider with a higher frequency RF system $(22.8 \mathrm{GHz})$ compared side by side with those for the drive beam necessary for the $11.4 \mathrm{GHz}$ upgrade. For this example it is necessary to assume an operating gradient for the $22.8 \mathrm{GHz}$ system, which we have taken to be 140 $\mathrm{MV} / \mathrm{m}$. Notice that the RF pulse length necessary for the two systems is a factor of two different. In the second column the drive beam has been created by increasing the drive beam linac length and energy and by adding another combiner ring to achieve a factor of 32 in compression. It is also assumed, of course that the deceleration and acceleration system as shown in Fig. 1 has been replaced by its $22.8 \mathrm{GHz}$ counterpart.

The interesting feature of this system, that is unique to two-beam systems, is that it is mostly unchanged. The drive beam linac is lengthened slightly, the combiner rings are reused and another is added. The entire drive beam distribution system is reused. This frequency flexibility allows one to envision a somewhat adiabatic transition to a two-beam linear collider using the experience at a lower frequency before tackling the more challenging problems at higher frequency.

Table 2: Drive Beam and Accelerator parameters for the 11.4 GHz Upgrade to $1.7 \mathrm{TeV}$ and for the $22.8 \mathrm{GHz}$ twobeam system. The $1.7 \mathrm{TeV}$ parameters include power from the conventional RF system.

\begin{tabular}{|l|r|r|}
\hline CM Energy (TeV) & 1.7 & 2.5 \\
\hline Average Gradient (MeV/m) & 78 & 118 \\
\hline Linac Length (Km) & 24 & 24 \\
\hline Repetition Frequency (Hz) & 60 & 60 \\
\hline Pulse Length (nsec) & 310 & 180 \\
\hline Number of bunches & 225 & 260 \\
\hline Charge per bunch (10^9) & 14 & 6.8 \\
\hline HE Beam Total Energy (KJ) & 410 & 360 \\
\hline Number of Drive Beams & 22 & 22 \\
\hline Rf Pulse Total Energy (KJ) & 780 & 900 \\
\hline Rf Pulse Length (nsec) & 450 & 225 \\
\hline Frequency Multiplication & 16 & 32 \\
\hline Deceleration Section Length (m) & 1070 & 1070 \\
\hline Drive beam Pulse (Microsec) & 160 & 160 \\
\hline Total Drive beam Energy (KJ) & 2100 & 2300 \\
\hline Drive Beam Energy (GeV) & 1.4 & 1.50 \\
\hline Drive Beam Current (A) & 10 & 10.0 \\
\hline Frequency of DBA (MHz) & 1428 & 1428 \\
\hline Length of DBA (m) & 230 & 250 \\
\hline Structure Length (m) & 1.7 & 1.70 \\
\hline Power per Structure (MW) & 100 & 100 \\
\hline Number of 50 MW Klystrons & 270 & 295 \\
\hline Total RF Efficiency (\%) & 40 & 40 \\
\hline Wall to beam Efficiency (\%) & 15 & 16.0 \\
\hline Wall Plug Power & 340 & 280 \\
\hline
\end{tabular}

Table 3: Parameters for upgrades to the NLC.

\begin{tabular}{|c|c|c|c|}
\hline Parameters & NLC & TB-NLC & TB-NLC \\
\hline CMS Energy $(\mathrm{GeV})$ & 1000 & 1700 & 2500 \\
\hline Luminosity $\left(10^{33}\right)$ & 34 & 94 & 99 \\
\hline Repetition Rate (Hz) & 120 & 60 & 60 \\
\hline Bunch Charge $\left(10^{10}\right)$ & 0.75 & 1.35 & 0.684 \\
\hline Bunches/RF Pulse & 190 & 190 & 260 \\
\hline Bunch Separation (ns) & 1.4 & 1.4 & 0.7 \\
\hline Eff. Gradient (MV/m & 50.2 & 78 & 118 \\
\hline Injected $\gamma \mathrm{e}_{\mathrm{x}} / \gamma \mathrm{e}_{\mathrm{y}}\left(10^{-8}\right)$ & $300 / 2$ & $300 / 2$ & $300 / 2$ \\
\hline$\gamma \mathrm{e}_{\mathrm{x}}$ at IP $\left(10^{-8} \mathrm{~m}-\mathrm{rad}\right)$ & 360 & 360 & 360 \\
\hline$\gamma \mathrm{e}_{\mathrm{y}}$ at IP $\left(10^{-8} \mathrm{~m}-\mathrm{rad}\right)$ & 3.5 & 3.5 & 3.5 \\
\hline$\beta_{\mathrm{x}} / \beta_{\mathrm{y}}$ at IP $(\mathrm{mm})$ & $10 / 0.12$ & $15 / 0.12$ & $6 / 0.06$ \\
\hline$\sigma_{x} / \sigma_{y}$ at IP (nm) & $190 / 2.1$ & $180 / 1.6$ & $94 / 0.93$ \\
\hline$\sigma_{z}$ at IP (um) & 110 & 110 & 50 \\
\hline Yave & 0.29 & 1.04 & 3.2 \\
\hline Pinch Enhancement & 1.49 & 1.6 & 1.5 \\
\hline Beamstrahlung dB (\%) & 10.2 & 31 & 33 \\
\hline Photons per e+/e- & 1.3 & 2.3 & 1.7 \\
\hline Linac Length $(\mathrm{km})$ & 12 & 12 & 12 \\
\hline
\end{tabular}

\section{SUMMARY}

In this paper we have discussed prospects for multi $\mathrm{TeV}$ linear colliders by giving specific examples of how the NLC might be upgraded in two steps. A two-beam upgrade to $1.7 \mathrm{TeV}$ using $11.4 \mathrm{GHz} \mathrm{RF}$ can be designed to permit a further upgrade using $22.8 \mathrm{GHz} \mathrm{RF}$ to 2.5 $\mathrm{TeV}$. These examples illustrate that the next generation linear collider can form the foundation for a multi $\mathrm{TeV}$ linear collider and can provide a platform for further exploration beyond $1 \mathrm{TeV}$.

\section{REFERENCES}

[1] H.H. Braun, et al., "A New Method of RF Power Generation for Two-Beam Linear Colliders", Proc. Of EPAC98, Stockholm, Sweden, June 1998.

[2] H.H. Braun, et al., "The CLIC RF Power Source, A Novel Scheme of Two-Beam Acceleration for $\mathrm{e}^{ \pm}$Linear Colliders", CERN 99-06.

[3] C. Adolphsen et al, ROAA003 in Proc. of PAC2001, Chicago, IL, June 2001, SLAC-PUB-8901.

[4] R.H. Miller et al, FPAH062 in Proc. of PAC2001, Chicago, IL, June 2001, SLAC-PUB-8897.

[5] Z. Li et al., FPAH061 in Proc. of PAC2001, Chicago, IL, June 2001, SLAC-PUB-9049.

[6] R. Jones et al, FPAH058 in Proc. of PAC2001, Chicago, IL, June 2001, SLAC-PUB-8889.

[7] R. Jones et al, FPAH068 in Proc. of PAC2001, Chicago, IL, June 2001, SLAC-PUB-8886.

[8] V. Dolgashev et al, FPAH067 in Proc. of PAC2001, Chicago, IL, June 2001, SLAC-PUB-8866.

[9] J. Irwin, J. Rifkin and R.D. Ruth, "A New Configuration for Two-Beam Linear Colliders", Proc. of the XX Int. Linac Conf., Monterey, CA, Aug. 2000, 782. 